\title{
THE EXISTENCE OF A SOLUTION FOR SEMI-LINEAR ABSTRACT DIFFERENTIAL EQUATIONS WITH INFINITE $B$-CHAINS OF THE CHARACTERISTIC SHEAF
}

\author{
A. Qazza ${ }^{1}$, R. Hatamleh ${ }^{2}$ \\ ${ }^{1}$ Department of Mathematics \\ Zarqa University \\ P.O. Box 132222, Zarqa, 13132, JORDAN \\ ${ }^{2}$ Department of Mathematics \\ Jadara University \\ P.O. Box 733, Irbid, 21110, JORDAN
}

\begin{abstract}
Initial-value problems for a semi-linear differential operator equations with singular linear part are considered. The existence of the infinite $B$-chains for the characteristic sheaf $\lambda A+B$ is assumed. In this case conditions for solvability have been obtained. The results are applied to a mixed problem for a partial differential equation.
\end{abstract}

AMS Subject Classification: $34 \mathrm{G} 20$

Key Words: infinite B-chains, singular operator sheaf, nonlinear differential equation, Frechet derivative

\section{Introduction}

We consider the initial-value problem for the semi-linear abstract differential equation

$$
\begin{gathered}
\frac{d(A u(t))}{d t}+B u(t)=f(t, u), \\
u(0)=u_{0},
\end{gathered}
$$

where $A, B$ are closed linear operators from a Hilbert $X$ space into a Hilbert space $Y$. In general the operator $A$ is not invertible. Semi-linear equations

Received: June 15, 2018

(c) 2018 Academic Publications

${ }^{\S}$ Correspondence author 
(1) with not invertible operator at the derivative in Banach spaces have been studied in works [1], [3], [4], [5]. The properties of the solution of initial-value problem (1), (2) are related to the properties of the characteristic $\lambda A+B$ sheaf of the equation's linear part (1). The operator sheaf $\lambda A+B$ is called regular when there is a complex number $\lambda_{0} \in \mathbb{C}$, for which the resolvent $\left(\lambda_{0} A+B\right)^{-1}$ is defined and is bounded on the subspace $D_{A} \cap D_{B}$. Otherwise, the sheaf $\lambda A+B$ is called singular.

The works [3] and [4] are based on the assumption that the characteristic sheaf $\lambda A+B$ is regular. In [4] the case of a singular characteristic sheaf $\lambda A+B$ is considered.

Definition 1.1. A pair of subspaces $\left(X^{\prime}, Y^{\prime}\right)$ is called an invariant relative to the sheaf $\lambda A+B$, if

$$
A\left(D_{A} \cap X^{\prime}\right) \subset Y^{\prime}, B\left(D_{B} \cap X^{\prime}\right) \subset Y^{\prime}
$$

and if at least one of the subspaces $X^{\prime}, Y^{\prime}$ is non-trivial.

Pairs of subspaces $\left(X_{1}, Y_{1}\right),\left(X_{2}, Y_{2}\right)$ reduce the sheaf $\lambda A+B$, if,

$$
X=X_{1} \dot{+} X_{2}, Y=Y_{1} \dot{+} Y_{2}, A\left(D_{A} \cap X_{i}\right) \subset Y_{i}, B\left(D_{B} \cap X_{i}\right) \subset Y_{i}, i=1,2 .
$$

In [5] the existence of a singular and a regular pair of reduced subspaces $\left(X_{s}, Y_{s}\right),\left(X_{r}, Y_{r}\right)$ is assumed:

$$
\begin{array}{r}
X=X_{S} \dot{+} X_{R}, Y=Y_{S} \dot{+} Y_{R}, \\
D_{A}=\left(D_{A} \cap X_{i}\right) \dot{+}\left(D_{A} \cap X_{R}\right), \\
D_{B}=\left(D_{B} \cap X_{S}\right) \dot{+}\left(D_{B} \cap X_{R}\right), \\
A\left(D_{A} \cap X_{s}\right) \subset Y_{s}, \quad B\left(D_{B} \cap X_{s}\right) \subset Y_{s}, \\
A\left(D_{A} \cap X_{r}\right) \subset Y_{r} \quad, \quad B\left(D_{B} \cap X_{r}\right) \subset Y_{r} .
\end{array}
$$

The sheaf of operators $\lambda A_{s}+B_{s}=\lambda A+\left.B\right|_{X_{S}}$ induced from the subspace $X_{S}$ into the subspace $Y_{S}$ is singular; the sheaf $\lambda A_{R}+B_{R}: X_{R} \rightarrow Y_{R}$ induced from the subspace $X_{R}$ into the subspace $Y_{R}$ is regular.

In [5] it was assumed that the structure of the singular component of the sheaf $\lambda A_{S}+B_{S}$ corresponds to a canonical form of the singular matrix sheaf according to L. Kroneker. Correspondingly, a canonical basis in the subspace $X_{S}$ exists, which consists of the L. Kroneker finite singular chains.

In [6] the infinite chains for the pairs of operators $A, B$ are introduced in connection with some problems of the perturbation theory for linear operator equations.

Definition 2.1. A sequence of vectors $\left\{x_{i}\right\}_{i=1}^{\infty} \subset D_{A} \cap D_{B}$ is called infinite $B$-chains for the sheaf $\lambda A+B$ if the vectors $\left\{x_{i}\right\}_{i=1}^{\infty}$ satisfy the following recurrent correlations 


$$
A x_{1}=0, \quad B x_{i}=A x_{i+1}, \quad i=1,2,3, \ldots,
$$

and if $\left\{B x_{i}\right\}_{i=1}^{\infty}$ are linearly independent vectors.

In this work the initial-value problem (1), (2) in the case of the singular characteristic sheaf $\lambda A+B$ with the infinite $B$-chains will be considered.

\section{Example. The Initial-Value Problem for a Partial Derivative Equation with Infinite $B$ - Chains of the Characteristic Sheaf $\lambda A+B$}

In the domain $t \geq 0,-\pi \leq x \leq \pi$ we shall consider a mixed problem

$$
\begin{gathered}
e^{-i x} \frac{\partial^{2} u(t, x)}{\partial t \partial x}-i e^{-i x} \frac{\partial u(t, x)}{\partial t}+\frac{\partial u(t, x)}{\partial x}=f(t, u), \\
u(t,-\pi)=u(t, \pi), \quad u(0, x)=u_{0}(x) .
\end{gathered}
$$

We shall assume that $f(t, u):[0, \tau] \times C \rightarrow C$ is a continuous complex function.

Let us introduce the following operators in the space $C_{[-\pi . \pi]}=X=Y$ :

$$
\begin{aligned}
& A=e^{-i x} \frac{d(.)}{d x}-i e^{-i x} \quad, \quad B=\frac{d(.)}{d x}, \\
& D_{A}=D_{B}=D=\left\{y \in C_{[-\pi, \pi]}^{1}: y(t,-\pi)=y(t, \pi)\right\} .
\end{aligned}
$$

Considering function $u(t, x)$ as a mapping $u(t):[0, \tau] \rightarrow C_{[-\pi, \pi]}^{1}$, let us represent the initial-value problem (5), (6) as an equivalent to the Cauchy abstract problem (1), (2) in the spaces $C_{[-\pi, \pi]}^{1}=X=Y$.

The characteristic sheaf $\lambda A+B$ is singular in fact, the equation

$$
(\lambda A+B) y=0
$$

can be rewritten in the form

$$
\left(\left(\lambda e^{-i x}+1\right) y\right)^{\prime}=0 .
$$

Clearly, in the case $|\lambda| \neq 1$ the equation (8) has a non-trivial solution - the function

$$
y_{0}(x, \lambda)=\left(\lambda e^{-i x}+1\right)^{-1} ;
$$

while in the case $|\lambda|=1$, the coefficient by the derivative of the differential operator $\lambda A+B$ is irreversible.

The sequence of exponents $\left\{e^{i k x}\right\}_{k=1}^{\infty}$ forms infinite $B$-chains for the sheaf $\lambda A+B$.

In fact, the exponents which are in the domain of the sheaf $\lambda A+B$ : $e^{i k x} \in C_{[-\pi, \pi]}^{1}, e^{-i k x}=e^{i k x}$ satisfies the correlation (4): 


$$
A\left(e^{i x}\right)=0, B\left(e^{i x}\right)=i e^{i x}=A\left(e^{i 2 x}\right), \ldots, B\left(\left(e^{i k x}\right)=k i e^{i k x}=A\left(\left(e^{i(k+1) x}\right), \ldots ;\right.\right.
$$

the sequence of functions $\left\{B x_{i}\right\}_{i=1}^{\infty}=\left\{e^{i k x}\right\}_{k=1}^{\infty}$ forms the system of linearly independent functions.

In the next section the initial-value problem (1), (2) in a general case of a characteristic sheaf with the infinite $B$-chains will be considered.

\section{The Theorem of Existence of a Solution of the Initial-Value Problem (1), (2) with the Infinite $B$-Chains for a Characteristic Sheaf}

The initial-value problem (1), (2) in the Hilbert spaces $X, Y$ is considered in this work. We assume that the decompositions (3), $D_{A} \subset D_{B}$, hold true; the regular component of the sheaf $\lambda A_{R}+B_{R}$ has the bounded inverse operator $A_{R}^{-1} \in L\left(Y_{R}, X_{R}\right)$,

Hence, $B_{R} A_{R}^{-1} \in L\left(Y_{R}\right)$.

Furthermore, let us assume that the following orthogonal decompositions hold true for the singular pair of the subspaces $\left(X_{S}, Y_{S}\right)(3)$

$$
X_{S}=\operatorname{ker} A \cap \operatorname{ker} B \oplus X_{1}, \quad, \quad Y_{S}=\operatorname{ker} A^{*} \cap \operatorname{ker} B^{*} \oplus Y_{1},
$$

where the subspace $X_{1}$ is the closing of the linear span of the finite set of the infinite $B$-chains, $Y_{1}=B\left(X_{1}\right)$.

The first vector for each of the $B$-chains belongs to the subspace ker $A$.

We shall denote the closing of the linear span of the other vectors by $X_{2}$.

From $A_{R}^{-1} \in L\left(Y_{R}, X_{R}\right)$ follows the decomposition $X_{S}=\operatorname{ker} A \dot{+} X_{2}$.

We introduce two pairs of the mutually complementary projectors

$$
\begin{aligned}
K & : \quad X \rightarrow \operatorname{ker} A, \quad P=(1-K): X \rightarrow X_{2} \dot{+} X_{R}, K^{2}=K \\
L & : Y \rightarrow \overline{\operatorname{Im} A}, \quad Q: Y \rightarrow \operatorname{ker} A^{*}, L^{2}=L .
\end{aligned}
$$

Definition 3.1. The continuous vector-function $u(t) \in C\left(\left[0, \tau_{0}\right], X\right)$ with the values from $D_{A} \cap D_{B}$ is called the solution of the equation (1) in the range $0 \leq t \leq \tau_{0}$, if $A u(t) \in C^{1}\left(\left[0, \tau_{0}\right], Y\right)$ and the function $u(t)$ satisfies the equation (1) for all $t \in\left[0, \tau_{0}\right]$.

Let us designate a closed sphere in the Hilbert space $X$ with the center $x_{0} \in X$ as $B\left(X, x_{0}, r\right)=\left\{x \in X:\left\|x-x_{0}\right\| \leq r\right\}$. 
Theorem 3.1. Let the decompositions (3),(10) hold true for the equation (1). Let $\operatorname{dim}\left(\operatorname{ker} A^{*} \cap \operatorname{ker} B^{*}\right) \leq \operatorname{dim} \operatorname{ker} A$ and vector $u_{0} \in D_{A}$ satisfies the condition $Q f\left(0, u_{0}\right)=0$.

Assume that the function $f(t, u)$ is continuous in the set $[0, \tau] \times B\left(X, u_{0}, r\right)$, the component $L f$ of the vector-function $f(t, u)$ satisfies the Lipschitz condition

$$
\begin{gathered}
\left\|L f\left(t, u^{1}\right)-L f\left(t, u^{2}\right)\right\| \leq a\left\|u^{1}-u^{2}\right\|, \quad \forall u^{1}, u^{2} \in B\left(X, u_{0}, r\right), \\
t \in[0, \tau],
\end{gathered}
$$

and the component $Q f$ has a continuous Frechet derivative $\frac{\partial Q f(t, u)}{\partial u}$.

If

$$
\left.\operatorname{rang} \frac{\partial}{\partial K u} Q f(0, u)\right|_{u=u_{0}}=\operatorname{dim}\left(\operatorname{ker} A^{*} \cap \operatorname{ker} B^{*}\right),
$$

then the problem (1),(2) has the solution $u(t)$ on the non-trivial interval $\left[0, \tau_{0}\right]$. Moreover the solution is unique if

$$
\operatorname{dim} \operatorname{ker} A=\operatorname{dim}\left(\operatorname{ker} A^{*} \cap \operatorname{ker} B^{*}\right) \text {. }
$$

Proof. Let us denote

$$
u_{K}=K u, u_{p}=P u, A_{L}=L A, B_{L}=L B .
$$

From the construction projector of $K$ follows that $K(X)=\operatorname{ker} A$.

This means that, $A K=0$.

From the other side of the definition of infinite $B$-chains it follows that $B(\operatorname{ker} A) \subset \operatorname{Im} A$.

Hence $Q B K=0$.

Therefore, equation (1) is equivalent to the following two equations

$$
\begin{gathered}
Q f\left(t, u_{k}+u_{p}\right)=0, \\
\frac{d\left(A_{L} u_{p}(t)\right)}{d t}+B_{L} u_{p}(t)=L f\left(t, u_{k}+u_{p}\right)-B_{L} u_{k}(t) .
\end{gathered}
$$

From the conditions of Theorem 3.1 it follows that there exist subspace $X_{10} \subset$ ker $A$ and the projector $K_{1}: X \rightarrow X_{10}$ such that $\operatorname{dim} X_{10}=\operatorname{dim} Y_{1}, K_{1} K=K$, and the linear operator $\frac{\partial}{\partial K_{1} u} Q f\left(0, u_{0}\right): X \rightarrow X_{10}$ is invertible.

Let us denote $K_{2}=K-K_{1}, X_{11}=K_{2}(X), u_{K_{1}}=K_{1} u_{K}, u_{K_{2}}=K_{2} u_{K}$. If we substitute $u_{K}=u_{K_{1}}+u_{K_{2}}$ in the equation (12) and apply the theorem of implicit function [2], we get the function

$$
u_{K_{1}}=\Psi\left(t, u_{K_{2}}, u_{p}\right) .
$$

This function has a continuous Frechet derivative $\frac{\partial \Psi}{\partial u_{p}}$ and defined on the set $\Omega=\left\{\left(t, u_{K_{2}}, u_{p}\right)\right\}=\left[0, \tau_{1}\right] \times B\left(X_{11} \dot{+} X_{2} \dot{+} X_{R},\left(K_{2}+P\right) u_{0}, r_{1}\right), \quad r_{1 \leq r}, \tau_{1 \leq \tau}$. 
Now changing $u_{p}=A_{L}^{-1} v$ and substitute function $u_{K_{1}}=\Psi\left(t, u_{K_{2}}, u_{p}\right)$ in the equation (13) we obtain

$$
\frac{d v}{d t}+B_{L} A_{L}^{-1} u_{p}(t)=\left(t, u_{K_{2}}, \nu\right)-B_{L} \Psi\left(t, u_{K_{2}}, \nu\right)-B_{L} u_{K_{2}},
$$

where

$$
\begin{aligned}
\varphi\left(t, u_{K_{2}}, \nu\right) & =L f(t, u), \Psi\left(t, u_{K_{2}}, \nu\right)=\Psi\left(t, u_{K_{2}}, A_{L}^{-1} v\right), \\
u & =\Psi\left(t, u_{K_{2}}, A_{L}^{-1} v\right)+u_{K_{2}}+A_{L}^{-1} v .
\end{aligned}
$$

Let us introduce the set

$$
G=\left[0, \tau_{2}\right] \times B\left(X_{11} \dot{+} X_{2} \dot{+} X_{R},\left(K_{2}+P\right) u_{0}, r_{1}\right), \quad r_{2} \leq r, \tau_{2 \leq} \tau_{1} .
$$

From the conditions of Theorem 3.1 it follows that the function $\Psi\left(t, u_{K_{2}}, \nu\right)$ satisfies the Lipschitz condition on $G$. We apply the Picard theorem [6] to the equation (14) with the initial condition $\nu(0)=A_{L} u_{p}(0)$. We obtain a continuous differentiable solution $v\left(t, u_{K_{2}}\right)$ on some set

$$
\Phi=\left\{\left(t, u_{k_{2}}\right)\right\}=\left[0, \tau_{0}\right] \times B\left(X_{11}, K_{2} u_{0}, r_{3}\right) .
$$

The obtained function defines a continuous differentiable function $u_{2}(t)=$ $A_{L}^{-1} v\left(t, u_{K_{2}}\right)$, which is the component of the solution $u(t)$.

The component $u_{K_{2}}$ is being chosen arbitrarily from the class $C\left(\left[0, \tau_{0}\right]\right.$; $B\left(X_{11}, K_{2} u_{0}, r_{3}\right)$ with the initial condition $K_{2} u(0)=K_{2} u_{0}$.

Finally, the function $u=\Psi\left(t, u_{K_{2}}, u_{p}\right)+u_{K_{2}}+u_{p}$ is the solution of the problem (1), (2).

In the mixed problem (5), (6) we consider the restriction of the operators $A, B(7)$ in a Hilbert spaces $X^{\prime}=Y^{\prime}=L_{[-\pi, \pi]}^{2}$.

Introduce the following subspaces

$$
\begin{gathered}
X_{S}=\overline{\operatorname{Lin}\left\{e^{i k x}\right\}_{k=1}^{\infty}}, Y_{S}=\overline{\operatorname{Lin}\left\{e^{i k x}\right\}_{k=0}^{\infty}}, X_{R}=\overline{\operatorname{Lin}\left\{e^{i k x}\right\}_{k=0}^{\infty}}, \\
Y_{R}=\overline{\operatorname{Lin}\left\{e^{i k x}\right\}_{k=1}^{\infty}}, \operatorname{ker} A=\left\{e^{i x}\right\}, X_{1}=X_{S}=Y_{1}, \\
X_{2}=\overline{\operatorname{Lin}\left\{e^{i k x}\right\}_{k=2}^{\infty}}, \operatorname{ker} A^{*} \cap \operatorname{ker} B^{*}=\operatorname{Lin}\{1\} .
\end{gathered}
$$

It may be shown that for $|\lambda|>1$ the operator $\left(\lambda A_{R}+B_{R}\right)$ is invertible bounded and defined on all subspace $Y_{R}$.

Note that for $|\lambda|>1$ the function $y_{0}(x, \lambda)(9)$ is expanded into the following series:

$$
y_{0}(x, \lambda)=\left(\lambda e^{-i x}+1\right)^{-1}=\frac{1}{\lambda} e^{i x}\left(1+\frac{1}{\lambda} e^{i x}\right)^{-1}
$$




$$
=\frac{1}{\lambda} e^{-i x}-\frac{1}{\lambda^{2}} e^{-i 2 x}+\ldots(-1)^{k-1} \frac{1}{\lambda^{k}} e^{-i k x}+\ldots,
$$

and for $|\lambda|<1$ the function $y_{0}(x, \lambda)(9)$ expanded as follows:

$$
y_{0}(x, \lambda)=\left(\lambda e^{-i x}+1\right)^{-1}=1-\lambda e^{-i x}+\ldots(-1)^{k} e^{-i k x}+\ldots \quad .
$$

The sequence of functions $\left\{e^{i k x}\right\}_{k=0}^{\infty}$ forms the infinite $A$-chains [6]:

$$
B(1)=0, A(1)=-i e^{-i x}=B\left(e^{-i x}\right), \ldots-i(k+1) e^{-i(k+1) x}=B\left(e^{-i(k+1) x}\right), \ldots
$$

Hence, if $|\lambda|<1$ then the complex number $\lambda$ is the singular point of the sheaf $\lambda A_{R}+B_{R}$.

We shall be obtain a representation for $A_{R}^{-1} \in L\left(Y_{R}, X_{R}\right)$. Let us show that for any function $f \in Y_{R}$ there exists a unique function $y \in X_{R} \cap Y_{R}$, so that $A_{R} y=f$.

Consider the equation $A_{R} y=f$ :

$$
e^{-i x} y^{\prime}-e^{-i x} y=f .
$$

This equation can be written in the form

$$
\left(e^{-i x} y\right)^{\prime}=f .
$$

From (15) it follows that:

$$
y=e^{i x} \int_{-\pi}^{\pi} f(s) d s+C e^{i x} .
$$

Let us prove that the function $Y(16)$ belong to the domain of the operator $A$.

Really, $y(-\pi)=-C$; on the other side, using $f \in Y_{R}$ we obtain:

$$
y(\pi)=-\int_{-\pi}^{\pi} f(s) d s-C=-(f, 1)-C=-C .
$$

We shall find the value $C$ so that the function $y$ (16) shall belongs to the subspace $X_{R}$.

Calculate the scalar product $\left(y, e^{i x}\right)$ :

$$
\left(y, e^{i x}\right)=\int_{-\pi}^{\pi} \int_{-\pi}^{x} f(s) d s d x+C \int_{-\pi}^{\pi} d x=\int_{-\pi}^{\pi} \int_{-\pi}^{x} f(s) d s d x+2 \pi C .
$$


Hence, for the function $y$ (16) belongs to the subspace $X_{R}$, it is necessary that $C$ has the form

$$
C=\frac{1}{2 \pi} \int_{-\pi}^{\pi} \int_{-\pi}^{x} f(s) d s d x
$$

Now we calculate the scalar product $\left(y, e^{i x}\right)$ for $k>1$, using the fact that $f \in Y_{R}:$

$$
\begin{aligned}
\left(y, e^{i k x}\right) & =\int_{-\pi}^{\pi} e^{-i(k-1) x} \int_{-\pi}^{\pi} f(s) d s d x+C \int_{-\pi}^{\pi} e^{-i(k-1) x} d x \\
& =-\left.\frac{i}{k-1} e^{-i(k-1) x} \int_{-\pi}^{\pi} f(s) d s\right|_{-\pi} ^{\pi}+\frac{i}{k-1} \int_{-\pi}^{\pi} e^{-i(k-1) x} f(x) d x \\
& =\frac{i}{k-1}\left((-1)^{k}(f, 1)+\left(f, e^{i(k-1) x}\right)\right)=0 .
\end{aligned}
$$

Thus, the representation for the operator $A_{R}^{-1}$ has the form:

$$
A_{R}^{-1} y=e^{i x}\left(\int_{-\pi}^{\pi} f(s) d s+\frac{1}{2 \pi} \int_{-\pi}^{\pi} \int_{-\pi}^{x} f(s) d s d x\right) .
$$

Calculate the projectors $Q$ and $K$ :

$$
Q y=\frac{1}{2 \pi} \int_{-\pi}^{\pi} y d x, \quad K y=\frac{1}{2 \pi} \int_{-\pi}^{\pi} y e^{-i x} d x . e^{i x} .
$$

Then the conditions of Theorem 3.1 for the mixed problem (5), (6) have the form:

1) $u_{0} \in D_{A} \Longleftrightarrow u_{0}(-\pi)=u_{o}(\pi)$;

2) $Q f\left(0, u_{0}\right)=0 \Longleftrightarrow \int_{-\pi}^{\pi} f\left(0, u_{0}\right) d x=0$;

3) $\frac{\partial f(t, u)}{\partial u}$ is continuous;

4) $\int_{-\pi}^{\pi} \frac{\partial f\left(0, u_{0}\right)}{\partial u} d x=0$.

For example, $f(t, u)=u^{2}-1$. 
Hence, there exists a single solution of the problem (5), (6) for any function $u_{0}(x)$ that satisfies the following conditions:

1. $u_{0}(-\pi)=u_{0}(\pi) ;$
2. $\int_{-\pi}^{\pi} u_{0}^{2}(x) d x=2 \pi ;$
3. $\int_{-\pi}^{\pi} u_{0}(x) d x \neq 0$.

For example, $u_{0}(x)=e^{i x}+1$.

\section{Conclusion}

In this work conditions for solvability of the Cauchy problem for some singular differential operator equations are received. In our case the singular characteristic sheaf $\lambda A+B$ has only infinite $B$-chains. If the characteristic sheaf $\lambda A+B$ has as infinite $B$-chains as finite singular chains, then the conditions for solvability will be other form. This problem will be considered in the next work.

The results have been applied to the investigation of mixed problems for partial differential equations. These mixed problems may be received in the investigation of the waveguide.

\section{Acknowledgements}

This research is funded by the Deanship of Research in Zarqa University, Jordan.

\section{References}

[1] R.W. Carroll, E. Showalter, Singular and Degenerate Cauchy Problems, Academic Press, New York - London (1976).

[2] J. Dieudonne, Foundations of Modern Analysis, Academic Press, New York - London (1960).

[3] A. Favini, P. Plazzi, Some results concerning the abstract nonlinear equation $D_{t} M u(t)+L u(t)=f(t, K u(t))$, Circuits Systems and Signal Processing, 5, No 2 (1986), 261-274. 
[4] A. Favini, A. Rutkas, Existence and uniqueness of solutions of some abstract degenerate nonlinear equations, Differential Integral Equations, 12, No 3 (1999), 373-394.

[5] P.S. Kabalyants, A.G. Rutkas, Two theorems on existence of solutions of a quasilinear singular differential-operator equation, Nonlinear Oscillations, 3, No 3 (2000), 358-365.

[6] M.M. Vainberg, V.A. Trenogin, Branching Theory of Solutions of Nonlinear Equations, Moscow, Nauka Publ. (1969) (in Russian). 